\title{
Hallazgos oculares como factores predictivos y diagnósticos de ehrlichiosis canina
}

\author{
Ocular findings as predictive and diagnostic factors of canine Erlichiosis \\ Dunia Yisela Trujillo Piso, ${ }^{1,2,3}$, Juan Sebastián Quijano Ramírez², \\ Mónica Yamile Padilla Barreto ${ }^{1,2}$, María del Pilar Sánchez Bonilla ${ }^{1,2}$
}

\section{Resumen}

\begin{abstract}
El objetivo del presente estudio fue determinar la posibilidad de predecir infecciones por Ehrlichia canis con base a lesiones oculares de caninos sin signos clínicos sistémicos aparentes sugerentes de infección por Erlichia. Se trabajó con 30 caninos llevados a la Clínica Veterinaria de la Universidad Cooperativa de Colombia (Ibagué) por alteraciones oculares. Se realizó el examen oftálmico completo. Aquellos con uveítis o hemorragias retinianas se les realizó el SNAP test para Ehrlichia canis. El principal motivo de consulta oftálmico de los pacientes positivos a $E$. canis fue secreción ocular (33.3\%), seguido ojo rojo $(30 \%)$ y de pérdida de la visión $(20 \%)$. Al examen oftálmico, la alteración ocular más frecuentemente fue disminución de la presión intraocular (PIO) $(93.3 \%)$ e hiperemia conjuntival (63.3\%). El 46.7\% presentó aumento de la secreción ocular y el $40 \%$ fue positivo a queratoconjuntivitis seca (KCS) (Rosa de Bengala + ). Desprendimiento de retina y edema perivascular de vasos retinianos fue encontrado en el $26.7 \%$ de los pacientes. En conclusión, el ojo rojo, PIO baja y la KCS de tipo cualitativo se presentan como hallazgos tempranos de la ehrlichiosis canina; por tanto, estos signos deberían ser considerados como sugerentes de la enfermedad en regiones endémicas.
\end{abstract}

Palabras clave: ojo rojo: Ehrlichia canis; desprendimiento de retina; uveítis; KCS; PIO

\footnotetext{
${ }^{1}$ Grupo de Investigación Impronta

${ }^{2}$ Facultad de Medicina Veterinaria y Zootecnia, Universidad Cooperativa de Colombia - Sede Ibagué, Colombia

${ }^{3}$ E-mail: dunia.trujillop@campusucc.edu.co

Fuente de financiación del estudio: Gobernación del Tolima y la Universidad de Ibagué en el marco del Convenio Especial de Cooperación N. ${ }^{\circ} 1026$ de 2013 «Desarrollo de una Cultura Científica en niños, niñas y jóvenes del departamento del Tolima» Línea B Semilleros de Investigación.
} 
The aim of this study was to determine the possibility of predicting Ehrlichia canis infections based on canine eye lesions without apparent systemic clinical signs suggestive of Erlichia infection. A total of 30 patient dogs taken to the Veterinary Clinic of the Cooperative University of Colombia (Ibagué) for ocular alterations were evaluated. A complete ophthalmic examination was performed. Those with uveitis or retinal hemorrhages were subjected to the SNAP test for Ehrlichia canis. The main reason for ophthalmic consultation of patients positive for E. canis was ocular secretion $(33.3 \%)$, followed by red eye (30\%) and loss of vision (20\%). In the ophthalmic examination, the most frequent ocular alteration was low intraocular pressure (IOP) (93.3\%) and conjunctival hyperemia (63.3\%). Moreover, $46.7 \%$ had an increase in ocular secretion and $40 \%$ were positive to keratoconjunctivitis sicca (KCS) (Rose Bengal +). Retinal detachment and perivascular edema of retinal vessels was found in $26.7 \%$ of patients. In conclusion, the red eye, low IOP and KCS of qualitative type are presented as early findings of canine ehrlichiosis; therefore, these signs should be considered as suggestive of the disease in endemic regions.

Key words: red eye; Ehrlichia canis; retinal detachment; uveitis; KCS; PIO

\section{INTRODUCCIÓN}

La Ehrlichia es una bacteria gramnegativa, pleomórfica, intracelular obligada que infecta a un amplio número de mamíferos. Se transmite a través de vectores como las garrapatas del género Ixodidae, particularmente el género Rhipicephalus y sus especies $R$. sanguineus y $R$. (B.) microplus (Paternina et al., 2009). La infección con E. canis da como resultado un amplio espectro de signos clínicos que van desde algunos clínicamente inaparentes hasta signos graves (Little, 2010).

Las alteraciones oculares son comunes en las infecciones por E. canis y se estima entre 15 y $100 \%$ de pacientes que pueden presentarlas (Leiva et al., 2005; Komnenou et al., 2007). Debido a su alta prevalencia, las afecciones oculares han venido siendo objeto de estudio en esta enfermedad, ya que pueden llevar a una pérdida total de la visión (Gould et al., 2000). La uveítis es el signo ocular más común en caninos afectados por ehrlichiosis. Células inflamatorias, principal- mente linfocitos y monocitos, son los principales causantes de la infiltración inflamatoria, lo que conlleva a la formación de complejos inmunitarios (Panciera et al., 2001). Los inmunocomplejos se depositan en los vasos sanguíneos locales, desequilibran las barreras oculares, aumentando el flujo de células inflamatorias en la úvea (Harrus et al., 2001; Gelatt, 2014).

Aunque las causas de la uveítis son diversas, Massa et al. (2002) demostró que el $17.6 \%$ son debidas a causas infecciosas siendo el agente causal más común la Ehrlichia canis. La uveítis no es solo un signo ocular importante en perros infectados con E. canis, pues se reporta, además, casos de desprendimiento de retina, conjuntivitis, petequias y equimosis en iris, edema de córnea, glaucoma secundario, neuritis óptica, hipema y hemorragias retinianas (Leiva et al., 2005; Komnenou et al., 2007; Wasik y Adkins, 2010).

El diagnóstico de ehrliquiasis puede hacerse empleando la citología para detección de mórulas de E. canis en el citoplasma de los monocitos en extendidos de sangre ente- 
ra, en frotis de capa blanca, en aspirados de bazo, médula ósea y nódulos linfáticos (Faria et al., 2010; Mylonakis et al., 2003); utilizando métodos serológicos como la inmunofluorescencia (IFA), considerada como la prueba serológica de oro (Harrus y Waner, 2011). No obstante, no permite diferenciar entre infecciones latentes o antiguas, dado que los títulos de anticuerpos se mantienen durante meses o años después del tratamiento y eliminación de la rickettsia (Harrus y Waner, 2011). Por otro lado, se dispone de la prueba de ELISA, través de kits comerciales (Harrus et al., 2002), donde se incluye al microorganismo completo como fuente antigénica (ImmunoComb ${ }^{\circledR}$ ) o a una parte del mismo como en los ensayos inmunocromatográficos (SNAP 4DX ${ }^{\circledR}$ - Anigen ${ }^{\circledR}$ E.canis/Anaplasma Ab Test Kit) (López et al., 2007).

La técnica de reacción en cadena de la polimerasa (PCR) y la secuenciación son las técnicas más sensibles para el diagnóstico y la caracterización de E. canis. La PCR cuantitativa o en tiempo real (RT PCR) es más sensible que la PCR convencional y permite la cuantificación de la carga bacteriana (copias/ $\mu 1)$. Se le ha utilizado para la cuantificación de carga ehrlichial en perros infectados de manera natural y experimental (Baneth et al., 2009). Por otro lado, las alteraciones hematológicas como trombocitopenia, anemia y leucopenia deben ser tenidas en cuenta como apoyo diagnóstico ante la sospecha de la enfermedad enfermedad (Das y Konar, 2013); de estos, la trombocitopenia es el hallazgo reportado con más frecuencia (Badillo et al., 2017; Kaewmongkol et al., 2017).

Las alteraciones oculares no han sido tomadas en cuenta como factores predictivos o con fines diagnósticos de la enfermedad, a pesar de que se reconoce la importancia de las uveítis y afecciones de las hemorrágicas oftálmicas en el curso de la enfermedad. Por esto, el objetivo del presente estudio fue determinar la posibilidad de predecir infeccio- nes por E. canis con base a lesiones oculares de caninos sin signos clínicos sistémicos aparentes,

\section{Materiales y Métodos}

Se incluyeron en el estudio 30 caninos sin raza, sexo o edad específica. Los animales eran pacientes que acudieron al servicio especializado de oftalmología veterinaria de la Clínica Veterinaria de la Universidad Cooperativa de Colombia (Ibagué) durante tres meses y fueron incluidos mediante un consentimiento informado por parte del propietario. El estudio fue aprobado por el Subcomité de Ética en Investigación de la Universidad Cooperativa de Colombia, seccional Bucaramanga, bajo el concepto 042-2018, siguiendo normas éticas de la declaración de Helsinki.

El motivo de consulta de los pacientes en todos los casos fue de alteración ocular sin aparente afección sistémica, por lo cual se sometieron a examen oftalmológico completo de ambos ojos. La valoración oftálmica se inició con la evaluación de la producción lacrimal (prueba de Schirmer), seguido de visualización con lámpara de hendidura y biomicroscopio para anexos y cámaras oculares. La integridad de la córnea se determinó a través de la prueba de fluoresceína y rosa de bengala. Para la evaluación de fondo de ojo se dilató la pupila con Tropicamida $1 \%$ (Mydriacil ${ }^{\circledR}$ ) y se empleó un oftalmoscopio indirecto 125 Welch Allyn ${ }^{\circledR}$ y un lente de 20 dioptrías. La toma de la presión fue realizada mediante tonometría deaplanación con tonómetro tipo Perkins (Kowa ${ }^{\circledR}$ modelo HA-2).

Los pacientes cuyos hallazgos oftálmicos sugirieron infección sistémica por Ehrlichia canis, se sometieron a punción venosa para extracción de sangre y posterior realización de la prueba Anigen $\operatorname{Rapid}{ }^{\circledR} E$. canis/Anaplasma Ab para detección del agente causal presuntivo. Todos los perros del estudio fueron positivos a esta prueba. Se 
utilizó estadística descriptiva para analizar los datos de los exámenes oculares.

\section{Resultados y Discusión}

De los 30 pacientes objeto de estudio, el $66.7 \%$ fue macho y el $33.3 \%$ hembra. A pesar de que la frecuencia de presentación de la enfermedad es mayor en machos, su prevalencia depende de factores propios de los individuos como su inmunidad o patogenicidad de la cepa causante de la infección y esta no varía con el sexo (da Silva et al., 2012; Nazari et al., 2013).

El rango de edad de los pacientes estuvo comprendido entre 0.5 y 9 años, con una mediana de 3 años (machos: 3.3; hembras: 4.7 años), lo cual coincide con otros estudios donde se estima que animales adultos y gerontes son los más acometidos por la bacteria, posiblemente debido a una mayor probabilidad de exposición al vector (RodriguezVivas et al., 2005; Nazari et al., 2013).

E1 53.3\% de los animales eran de raza pura, mientras que el $47 \%$ correspondió a mestizos. Se identificaron ejemplares de raza Labrador, Boxer, Bulldog, Pug, Golden Retriever, Pastor Alemán, Pastor Belga, Bull Terrier, Pitbull, Poodle, Weimaraner y Yorkshire Terrier. Estos resultados indican una escasa o nula asociación racial con la probabilidad de sufrir la infección (Hoyos et al., 2007; Huerto-Medina y Damaso, 2015), pero que contradicen los postulados que sugieren que el Pastor Alemán es la raza de mayor riesgo para adquirir la enfermedad (Niyindo et al., 1980). Cabe resaltar que los caninos objeto de estudio eran provenientes de hogares de condición socioeconómica media-baja y cuyos recursos limitan la compra de caninos de razas altamente valoradas económicamente y de porte grande.

La alteración ocular anormal más notada por los propietarios y que motivaron la búsqueda de una consulta oftálmica fue la

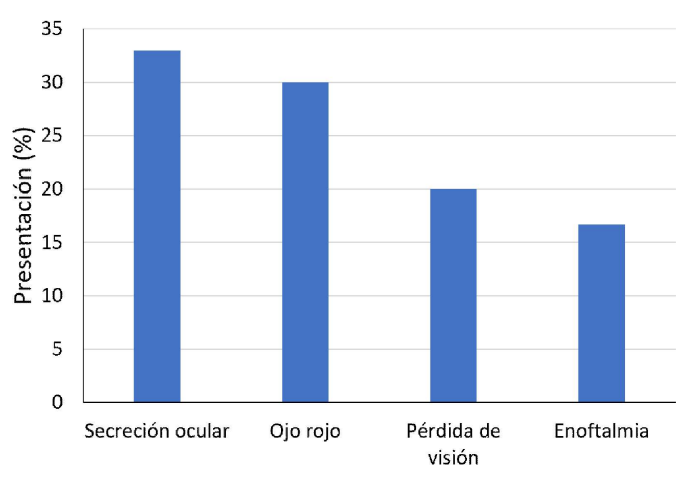

Figura 1. Principales motivos de consulta oftalmológica de pacientes caninos positivos a Ehrlichia canis

secreción ocular (33.3\%), seguida de ojo rojo (30\%), pérdida aguda de la visión $(20 \%)$ y enoftalmos (16.7\%) (Figura 1). La secreción ocular no es un signo común en la uveítis (Gwinn, 1988), pero su manifestación junto a la alta prevalencia del ojo rojo puede estar asociada a la presencia en la conjuntiva de células inflamatorias como macrófagos o neutrófilos, a consecuencia de la presencia de E. canis en este tejido; asimismo, la reacción inflamatoria que provoca puede interferir con la producción de la porción mucosa de la lágrima por parte de las células caliciformes (Baneth et al., 2009; WalserReinhardt et al., 2012).

Pérdida de la visión y enoftalmos se presentan con mayor frecuencia en pacientes con ehrlichiosis canina como signos de uveítis en la que ocurre hipotonía ocular, hemorragia de retina que cursa con desprendimiento o hemorragia de retina y disminución de la presión intraocular (Gelatt, 2014). Algunos pacientes presentan ptisis bulbi que podría estar relacionada a estos casos, aunque su presentación está asociada a cronicidad (Gelatt, 2014). Otros motivos de consulta adicionales al cuadro oftálmico principal incluyeron opacidad ocular, blefaritis, blefaroespasmo, melanosis corneal, anisocoria, hidroftalmía, edema de córnea, alopecia palpebral, opaci- 
Cuadro 1. Frecuencia (\%) de alteración de las estructuras oculares de pacientes positivos a Ehrlichia canis, pero sin signos clínicos de ehrlichiosis

\begin{tabular}{lccc}
\hline \multirow{2}{*}{ Estructura } & \multicolumn{3}{c}{ Resultado del examen oftalmológico } \\
\cline { 2 - 4 } & Normal & Alteración & No visualizado \\
\hline Globo ocular & 73.3 & 26.7 & 0 \\
Párpados & 63.3 & 36.6 & 0 \\
Conjuntiva & 10.0 & 90.0 & 0 \\
Córnea & 40.0 & 60.0 & 0 \\
Iris & 70.0 & 30.0 & 0 \\
Pupila & 63.3 & 36.7 & 0 \\
Cámara anterior & 80.0 & 20.0 & 0 \\
Fondo de ojo y vítreo & 30.0 & 20.0 & 50.0 \\
\hline
\end{tabular}

dad corneal, iris bombé y neovascularización corneal.

La presión intraocular promedio de los pacientes examinados fue de $8.0 \pm 3.6$ $\mathrm{mmHg}$, valor indicativo de presión intraocular (PIO) baja y sugerente de uveítis anterior en el $93.3 \%$ de los pacientes evaluados, por lo que la reducción de la PIO podría ser considerada como indicador de diagnóstico temprano de uveítis por E. canis. La PIO baja es el resultado de la presencia de complejos inmunes en las paredes de los vasos sanguíneos del tracto uveal que promueve aumento de la permeabilidad vascular y desequilibrio de las barreras oculares, lo que a su vez permite la afluencia de células inflamatorias mononucleares al ojo, acentuando las lesiones en el tracto uveal (Harrus et al., 2001). De otro lado, la activación de la cascada de ácido araquidónico asociada a la lesión de las membranas celulares, resulta en un aumento en las concentraciones de prostaglandinas, tromboxanos y leucotrienos (Harrus et al., 2001; Veloso et al., 2018), metabolitos que también estimulan la infiltración de las células inflamatorias en la úvea. Las prostaglandinas son potentes mediadores de la inflamación intraocular que pueden causar miosis por acción directa sobre el es- fínter del iris, hiperemia, y alteraciones en la PIO (Wasik y Adkins, 2010).

Los valores del test lacrimal de Schirmer (TLS) fueron de $18 \mathrm{~mm}$ en promedio, lo que indica que no había alteración en la producción acuosa de la lágrima de la lágrima en pacientes positivos a E. canis. Tampoco se encontró diferencia entre sexos para los valores de la PIO y el TLS ( $p=0.48$ y $p=0.61$, respectivamente) ni entre razas.

En el examen oftálmico se encontró que el globo ocular del $73.3 \%$ de los pacientes se encontraba normal, mientras que el restante $26.6 \%$ presentó alteraciones bulbares tales como exoftalmia, enoftalmia y hidroftalmía. Dentro de las alteraciones oculares que revistieron alto porcentaje de presentación se destaca la hiperemia conjuntival $(90 \%)$, cuya causa puede atribuirse a la presencia del agente $E$. canis en la conjuntiva (Baneth et al., 2009; Walser-Reinhardt et al., 2012). Asimismo, también fueron encontradas petequias, folículos, melanosis, quemosis y vasos epiesclerales.

Las alteraciones de córnea encontradas en el $60 \%$ de los pacientes fueron precipitados queráticos, vascularización y edema, de- 
mostrando que en la uveítis anterior, patógenos como E. canis originan inflamación intraocular que aumenta la permeabilidad del endotelio, disminuye la función de la bomba endotelial de sodio y potasio, reduce la densidad celular y aumenta el pleomorfismo y polimegatismo (Pillai et al., 2000). Precipitados queráticos ocurren como resultado de agregados de células inflamatorias que se adhieren al endotelio corneal (Cassin y Rubin, 2001).

Alteraciones palpebrales y pupilares fueron encontradas en igual porcentaje de frecuencia (36.7\%, Cuadro 1). Las primeras no constituyen hallazgos frecuentes en pacientes con E. canis y están asociadas a factores conformacionales (Maggs et al., 2013), mientras que las alteraciones pupilares se relacionan con uveítis inducida por E. canis, $\mathrm{y}$ de manera predominante se caracterizaron como miosis, afección altamente relacionada con la disminución de la presión intraocular derivada del efecto de las prostaglandinas (Wasik y Adkins, 2010).

Hallazgos del fondo de ojo, cámara anterior y vítreo en el presente estudio corroboran los postulados de la uveítis como manifestación ocular de infección sistémica por E. canis (Oriá et al., 2004). Se destaca que el $46 \%$ de los caninos presentaron edema perivascular, hemorragia y desprendimiento, neuritis óptica y vasos retinianos tortuosos; asimismo, el 30\% presentaron fondo y vítreo normal y en el $50 \%$ de los pacientes no fue posible observarlo. Las alteraciones de cámara anterior incluyeron llamarada acuosa $\mathrm{e}$ hifema (Cuadro 1).

En la prueba de fluoresceína, el 97\% de los animales fueron negativos, mientras que el $40 \%$ fue positivo a la prueba de rosa de bengala. Este hallazgo en conjunto con el motivo de la consulta médica relatado como aumento de secreción ocular e hiperemia conjuntival sugiere que pacientes con ehrlichiosis presentan queratoconjuntivitis seca de tipo cualitativo debido a la alta capacidad del agente causal de instalarse en el tejido conjuntival (Baneth et al., 2009; WalserReinhardt et al., 2012).

\section{Conclusiones}

- La disminución temprana de la PIO podría ser tomada en cuenta como factor predictivo de la presencia de ehrlichiosis canina en ausencia de signos clínicos sistémicos en áreas endémicas.

- Queratoconjuntivitis seca de tipo cualitativo debiera ser considerada como manifestación ocular temprana de ehrlichiosis canina.

\section{Literatura Citada}

1. Badillo M, Díaz A, Orozco C, Lavalle R. 2017. Infection by Ehrlichia canis and Anaplasma sp in dogs attended in veterinary clinics, Barranquilla, Colombia. Rev MVZ Córdoba 22: 6023-6033.

2. Baneth G, Harrus S, Ohnona FS, Schlesinger $Y$. 2009. Longitudinal quantification of Ehrlichia canis in experimental infection with comparison to natural infection. Vet Microbiol 136: 321325. doi: 10.1016/j.vetmic.2008.11.022

3. Cassin B, Rubin M. 2001. Dictionary of eye terminology. $4^{\text {th }}$ ed. Gainesville. Florida: Triad Publishing Company. $286 \mathrm{p}$.

4. Das M, Konar S. 2013. Clinical and hematological study of canine Ehrlichiosis with other hemoprotozoan parasites in Kolkata, West Bengal, India. Asian Pac J Trop Biomed 3: 913-915. doi: 10.1016/S2221-1691(13)60178-1

5. da Silva GC, Benitez N, Girotto A, Taroda A, Vidotto MC, Garcia JL, de Freitas JC, et al. 2012. Occurrence of Ehrlichia canis and Anaplasma platys in household dogs from northern Parana. Rev Bras Parasitol V 21: 379-385. doi: 10.1590/S1984-29612012005000009

6. Faria JL, Dagnone A, Munhoz T, João C, Biscola WA, Machado R, Tinucci-Costa M. 2010. Ehrlichia 
canis morulae and DNA detection in whole blood and spleen aspiration samples. Rev Bras Parasitol V 19: 98102. doi: $10.4322 / \mathrm{rbpv} .01902006$

7. Gelatt KN. 2014. Canine anterior uvea: diseases and surgery. In: Gelatt KN (ed). Essentials of veterinary ophthalmology. $3^{\text {rd }}$ ed. USA: John Wiley \& Sons. p 276-300.

8. Gould DJ, Murphy K, Rudorf H, Crispin M. 2000. Canine monocytic ehrlichiosis presenting as acute blindness 36 months after importation into the UK. J Small Anim Pract 41: 263-265. doi: 10.1111/j.1748-5827.2000.tb03937.x

9. Gwinn RM. 1988. Anterior uveitis: diagnosis and treatment. Semin Vet Med Surg 3: 33-39.

10. Harrus S, Waner T. 2011. Diagnosis of canine monocytotropic ehrlichiosis (Ehrlichia canis): an overview. Vet J 187: 292-296. doi: 10.1016/j.tvj1.2010.02.001

11. Harrus S, Alleman AR, Bark H, Mahan SM, Waner T. 2002. Comparison of three enzyme-linked immunosorbant assays with the indirect immunofluorescent antibody test for the diagnosis of canine infection with Ehrlichia canis. Vet Microbiol 86: 361-368. doi: 10.1016/S0378-1135(02)00022-6

12. Harrus S, Day MJ, Waner T, Bark H. 2001. Presence of immune-complexes, and absence of antinuclear antibodies, in sera of dogs naturally and experimentally infected with Ehrlichia canis. Vet Microbiol 83: 343-349. doi: 10.1016/ S0378-1135(01)00431-X

13. Hoyos L, Li O, Alvarado A, Suárez F, Díaz D. 2007. Evaluación del examen hematológico en el diagnóstico de ehrlichiosis canina. Rev Inv Vet Perú 18: 133139. doi: 10.15381/rivep.v18i2.1288

14. Huerto-Medina E, Dámaso-Mata B. 2015. Factores asociados a la infección por Ehrlichia canis en perros infestados con garrapatas en la ciudad de Huánuco, Perú. Rev Peru Med Exp Salud Pública 32: 756-760.
15. Kaewmongkol G, Lukkana N, Yangtara S, Kaewmongkol S, Thengchaisri N, Sirinarumitr T, Jittapalapong S, et al. 2017. Associa-tion of Ehrlichia canis, hemotropic Mycoplasma spp and Anaplasma platys and severe anemia in dogs in Thailand. Vet Microbiol 201: 195-200. doi: 10.1016/ j.vetmic.2017.01.022

16. Komnenou AA, Mylonakis ME, Kouti V, Tendoma L, Leontides L, Skountzou E, Dessiris A, et al. 2007. Ocular manifestations of natural canine monocytic ehrlichiosis (Ehrlichia canis): a retrospective study of 90 cases. Vet Ophthalmol 10: 137-142. doi: 10.1111/ j.1463-5224.2007.00508.x

17. Leiva M, Naranjo C, Peña MT. 2005. Ocular signs of canine monocytic ehrlichiosis: a retrospective study in dogs from Barcelona, Spain. Vet Ophthalmol 8: 387393. doi: $10.1111 / j .1463-5224.2005$.00409. $\mathrm{x}$

18. Little SE. 2010. Ehrlichiosis and anaplasmosis in dogs and cats. Vet Clin NAm-Small 40: 1121-1140. doi: 10.1016/ j.cvsm.2010.07.004

19. López L, Venteo A, Aguirre E, García $M$, Rodriguez J, Amusátegui I, Tesouro MA, et al. 2007. Development of a sensitive and specific indirect enzyme-linked immunosorbent assay based on a baculovirus recombinant antigen for detection of specific antibodies against Ehrlichia canis. J Vet Diagn Invest 642: 635-642. doi: 10.1177/ 104063870701900604

20. Maggs D, Miller P, Ofri R. 2013. Slatter's fundamentals of veterinary ophthalmology. $5^{\text {th }}$ ed. Elsevier. $520 \mathrm{p}$.

21. Massa KL, Gilger BC, Miller TL, Davidson MG 2002. Causes of uveitis in dogs: 102 cases (1989-2000). Vet Ophthalmol 5: 93-98.

22. Mylonakis ME, Koutinas AF, Billinis $C$, Leontides LS, Kontos $V$, Papadopoulos O, Rallis T, et al. 2003. Evaluation of cytology in the diagnosis of acute canine monocytic ehrlichiosis 
(Ehrlichia canis): a comparison between five methods. Vet Microbiol 91: 197-204. doi: 10.1016/S0378-1135(02)00298-5

23. Nazari M, Lim SY, Watanabe M, Sharma RSK, Cheng NA, Watanabe M. 2013. Molecular Detection of Ehrlichia canis in dogs in Malaysia. Plos Neglect Trop D 7: e1982. doi: 10.1371/ journal.pntd.0001982

24. Nyindo M, Huxsoll DL, Ristic M, Kakoma I, Brown JL, Carson CA, Stephenson EH. 1980. Cell-mediated and humoral immune responses of german shepherd dogs and beagles to experimental infection with Ehrlichia canis. Am J Vet Res 41: 250-254.

25. Oriá AP, Pereira PM, Laus JL. 2004. Uveitis in dogs infected with Ehrlichia canis. Cienc Rural 34: 1289-1295. doi: 10.1590/S0103-84782004000400055

26. Panciera RJ, Ewing SA, Confer AW. 2001. Ocular histopathology of Ehrlichial infections in the dog. Vet Pathol 38: 43-46. doi: 10.1354/vp.38-1-43

27. Paternina LE, Diaz-Olmos Y, Paternina-Gómez M, Bejarano E. 2009. Canis familiaris, un nuevo hospedero de Ornithodoros (A.) puerto- ricensis Fox, 1947 (Acari: Ixodida) en Colombia. Acta Biol Colomb 14: 154-160.

28. Pillai CT, Dua HS, Azuara-Blanco A, Sarhan AR. 2000. Evaluation of corneal endothelium and keratic precipitates by specular microscopy in anterior uveitis. Brit J Ophthalmol 84: 1367-1371. doi: 10.1136/bjo.84.12.1367

29. Rodriguez-Vivas RI, Albornoz REF, Bolio GEM. 2005. Ehrlichia canis in dogs in Yucatán, Mexico: seroprevalence, prevalence of infection and associated factors. Vet Parasitol 127: 8185. doi: 10.1016/j.vetpar.2004.08.022

30. Veloso J, Sauer L, Oriá A, Gomes D, Santos AC, Andrade CF, de Andrade TN, et al. 2018. Molecular diagnosis of Ehrlichia canis infection in dogs with uveitis. Ciências Agrárias 39: 1049-1056. doi: 10.5433/1679-0359.2018v39n3p1049

31. Walser-Reinhard L, SchaarschmidtKiener D, Forster JL, Matheis F, Spiess B. 2012. Direct detection of Ehrlichia canis by PCR in the conjunctiva of a dog with bilateral anterior uveitis. Schweiz Arch Tierh 154: 149152. doi: 10.1024/0036-7281/a000318

32. Wasik B, Adkins E. 2010. Canine anterior uveitis. Compend Contin Educ Vet 32(11): E1. 